\title{
Uniquely Hominid Features of Adult Human Astrocytes
}

\author{
Nancy Ann Oberheim, ${ }^{1,5}$ Takahiro Takano, ${ }^{1}$ Xiaoning Han, ${ }^{1}$ Wei He, ${ }^{1}$ Jane H. C. Lin, ${ }^{2}$ Fushun Wang, ${ }^{1}$ Qiwu Xu, ${ }^{1}$ \\ Jeffrey D. Wyatt, ${ }^{3}$ Webster Pilcher, ${ }^{1}$ Jeffrey G. Ojemann, ${ }^{4}$ Bruce R. Ransom, ${ }^{5}$ Steven A. Goldman, ${ }^{1}$ and \\ Maiken Nedergaard ${ }^{1}$ \\ ${ }^{1}$ Center for Translational Neuromedicine, Departments of Neurology and Neurosurgery, University of Rochester Medical Center, Rochester, New York \\ 14642, ${ }^{2}$ Department of Pathology, New York Medical College, Valhalla, New York 10595, ${ }^{3}$ Department of Comparative Medicine, University of Rochester, \\ Rochester, New York 14642, and Departments of ${ }^{4}$ Neurosurgery and ${ }^{5}$ Neurology, University of Washington, Seattle, Washington 98195
}

Defining the microanatomic differences between the human brain and that of other mammals is key to understanding its unique computational power. Although much effort has been devoted to comparative studies of neurons, astrocytes have received far less attention. We report here that protoplasmic astrocytes in human neocortex are 2.6-fold larger in diameter and extend 10-fold more GFAP (glial fibrillary acidic protein)-positive primary processes than their rodent counterparts. In cortical slices prepared from acutely resected surgical tissue, protoplasmic astrocytes propagate $\mathrm{Ca}^{2+}$ waves with a speed of $36 \mu \mathrm{m} / \mathrm{s}$, approximately fourfold faster than rodent. Human astrocytes also transiently increase cystosolic $\mathrm{Ca}^{2+}$ in response to glutamatergic and purinergic receptor agonists. The human neocortex also harbors several anatomically defined subclasses of astrocytes not represented in rodents. These include a population of astrocytes that reside in layers 5- 6 and extend long fibers characterized by regularly spaced varicosities. Another specialized type of astrocyte, the interlaminar astrocyte, abundantly populates the superficial cortical layers and extends long processes without varicosities to cortical layers 3 and 4 . Human fibrous astrocytes resemble their rodent counterpart but are larger in diameter. Thus, human cortical astrocytes are both larger, and structurally both more complex and more diverse, than those of rodents. On this basis, we posit that this astrocytic complexity has permitted the increased functional competence of the adult human brain.

\section{Introduction}

Recent studies of glial cell have produced a wealth of information regarding their functional roles and structural organization. However, these studies have been based primarily on observations in the rodent brain. It is generally assumed that human brain shares a multitude of similarities with rodent and that studies in mice and rats can serve as a model for similar measures in humans. However, it is essential to evaluate the similarities and differences between human brain and our experimental models.

Study of human glia dates back to 1858 when the concept of "Nervenkitt" or neuroglia was first introduced by Rudolf Virchow (Kettenmann and Verkhratsky, 2008). Although Virchow believed that neuroglia was primarily connective tissue, he described cellular elements within this nerve glue. Late in the 19th century, astrocytes were defined by Von Lenhossek and classified as either protoplasmic or fibrous based on morphology by Kolliker and Andrizen (Kettenmann and Verkhratsky, 2008). Ramon y Cajal studied the morphology of astrocytes within human and other mammalian brains (Ramon y Cajal, 1897). Although Ramon y Cajal's drawings depict the complexity of human astro-

\footnotetext{
Received Sept. 30, 2008; revised Jan. 13, 2009; accepted Jan. 24, 2009.

This work was supported by the G. Harold and Leila Y. Mathers Charitable Foundation and grants from the National Institute of Neurological Disorders and Stroke (M.N., S.A.G., B.R.R.).

Correspondence should be addressed to Dr. Maiken Nedergaard, Division of Glial Disease and Therapeutics, Center for Translational Neuromedicine, University of Rochester Medical Center, 601 Elmwood Avenue, Rochester, NY 14642.E-mail: nedergaard@urmc.rochester.edu.

DOI:10.1523/JNEUROSCI.4707-08.2009

Copyright $\odot 2009$ Society for Neuroscience $\quad$ 0270-6474/09/293276-12\$15.00/0
}

cytes, it was more recently appreciated that human and rodent astrocytes differ. A comparative study of Colombo and coworkers revealed that interlaminar astrocytes are primate-specific cells (Colombo et al., 1995; Colombo and Reisin, 2004). Interlaminar astrocytes were originally described by Andriezen and Retzius as small glial cells, which reside in the upper cortical layers, and extend long glial fibrillary acidic protein-positive $\left(\mathrm{GFAP}^{+}\right)$processes through cortical layers 2-4 (Andriezen, 1893; Retzius, 1894). The existence of millimeter long glial processes in primate cortex is distinctly different from the evenly spaced uniform astrocytes in rodent cortex. Nonetheless, a comprehensive analysis of human versus rodent astrocytes has to date been performed, despite numerous studies on the evolutionary changes of neurons (Nimchinsky et al., 1999; DeFelipe et al., 2002; Yanez et al., 2005; Prabhakar et al., 2006). The aim of this study was thus to directly compare human and rodent cortical astrocytes.

By immunostaining thick sections of freshly resected surgical samples of human temporal neocortex for GFAP and then using confocal imaging to follow and reconstruct single cells, we found that human astrocytes are significantly larger and structurally more complex than their rodent counterparts. Furthermore, human brain contains multiple classes of GFAP-immunoreactive cells with distinct morphological features, in particular, human brain is characterized by a novel astrocytic phenotype that we designate varicose projection astrocytes. These cells extend 1- to 5 -mm-long projection fibers that harbor regularly spaced varicosities. In addition to their morphological specializations, we noted that functionally, human astrocytes exhibited increased intracellular $\mathrm{Ca}^{2+}$ responses to purinergic and metabotropic re- 
ceptor agonists and were able to support $\mathrm{Ca}^{2+}$ waves at velocities significantly greater than in the rodent.

\section{Materials and Methods}

Tissue. Human brain tissue was acquired from a total of 30 patients during surgical resection for a variety of pathologies, including medically refractory epilepsy $(n=23)$, tumor $(n=6)$, and decompression attributable to abscess $(n=1)$. The age range of patients was $4-69$ years old, with a mean of 32.4 years. Only tissues dissected beyond the primary area of pathology were used. Adult primate cortical brain tissue was taken from a perfusion-fixed adult squirrel monkey [4\% paraformaldehyde (PFA)], two perfusion-fixed adult rhesus macaques, and a Formalinfixed adult common chimpanzee (Pan troglodytes). The chimpanzee died at 55 years of age from a cardiac condition. Adult FVB/NJ mice and Sprague Dawley rats (The Jackson Laboratory) were used as rodent comparisons. All experiments using human tissues were approved by the University of Rochester Research Subjects Review Board and the University of Washington Research Subjects Review Board; all animal studies were approved by the Institutional Animal Care and Use Committees of both the University of Rochester and the University of Washington.

Immunofluorescence. Human tissue from surgical resection was immediately placed in PBS containing 4\% PFA and fixed overnight. It was then washed in PBS for 4-6 h. Rhesus macaque monkeys, the squirrel monkey, mice, or rats were deeply anesthetized with a mixture of ketamine $(60 \mathrm{mg} / \mathrm{kg}$, i.p.) and xylazine (10 mg/kg, i.p.) and fixed in situ through perfusion of $4 \%$ PFA in PBS. Brains were removed and washed overnight in PBS at $4^{\circ} \mathrm{C}$. For additional examination of primates and for examination the vasculature in rodents, brains were acutely isolated and immediately placed in $4 \%$ PFA, or Formalin in the case of the chimpanzee brain, and fixed a minimum of $12 \mathrm{~h}$, similar to processing of the human surgical resections. After washing, all tissue was sectioned at 200-400 $\mu \mathrm{m}$ using a vibratome and immersed in PBS at $4^{\circ} \mathrm{C}$. Sections were then incubated in 5\% normal donkey serum (NDS) (Jackson ImmunoResearch) and $0.5-1 \%$ Triton X-100 (Sigma-Aldrich) for $1 \mathrm{~h}$. Sections were incubated in primary antibody in $1 \%$ NDS and $0.1-0.5 \%$ Triton X-100 overnight at $4^{\circ} \mathrm{C}$. Primary antibodies used were monoclonal anti-GFAP (mAB360, 1:500; Millipore) and polyclonal chicken anti-MAP2 (ab5392, 1:5000; Abcam). Sections were then washed and incubated in secondary antibodies (Jackson ImmunoResearch) and counterstained with 4,6diamindino-2-phenylindole (DAPI) (D-21490, 1:10,000; Invitrogen) or sytox (S7020, 1:5000; Invitrogen). Images were collected with a confocal laser-scanning microscope (FV500 or FV1000; Olympus) (Wang et al., 2004). For immunoelectron microscopy, GFAP and aquaporin-4 antibodies (AB3068; Millipore) were used, and sections were prepared from human tissue directly from surgical resection as described previously (Goldman and Nedergaard, 1992). Images were collected with an Hitachi 7100 electron microscope and analyzed with NIH ImageJ.

Diolistic labeling. The Helios Gene Gun System (165-2431; Bio-Rad) was used to deliver gold $(1.0 \mu \mathrm{m})$ and tungsten particles coated with lipophilic dyes, DiI (D282; Invitrogen) or DiD (D307; Invitrogen), into lightly fixed brain slices from surgical resection and perfusion-fixed rodent brain. Preparations of the DiOlistic bullets were based on the method of Gan et al. (2000) and Benediktsson et al. (2005). Two hundred to $400-\mu \mathrm{m}$-thick fixed brain slices were placed on $2 \%$ agarose blocks in a culture dish and shot with both DiI and DiD bullets using 100 psi of helium pressure. Slices were counterstained with sytox and mounted for imaging. When immunofluorescence was combined with diolistic labeling, immunofluorescence was performed first, without Triton X-100, and then, after secondary antibody incubation, the sections were shot with DiI and DiD bullets and counterstained.

Image collection and analysis. Images were collected with 20 and $40 \times$ oil objective lenses (numerical aperture 0.75 and 1.3 , respectively) with FluoView (Olympus) software and analyzed with both Fluoview and NIH ImageJ software. Stacks of $1 \mu \mathrm{m}$ inclement depth were used for analysis. Diameter of the cell was defined as the longest axis through the nucleus of the domain of the cell based on GFAP immunostaining. The thickness of the GFAP-positive processes was defined as the diameter of GFAP measured between two branching points, and the length of longest process was defined as the distance from the center of the nuclei to the tip of the extended GFAP-positive processes. The domain of the cell based on diolistic labeling was determined by the compilation of each extension of the diolistically labeled processes. Overlapping area of two neighboring astrocytic domains over $101-\mu \mathrm{m}$ stacks was averaged (Ogata and Kosaka, 2002). As a second measure of overlap, processes that trespassed into the domain of the adjacent astrocyte were traced to obtain each length. The summation of the lengths of these processes over $101-\mu \mathrm{m}$ stacks was also averaged to determine the degree of the domain organization (Oberheim et al., 2008). The means, SDs, and SEMs were calculated, and the differences between groups were tested with a Student's $t$ test or ANOVA with Bonferroni's post hoc test, with significance determined to occur when $p<0.05$ using a two-tailed test.

Calcium imaging. Human tissue was prepared similar to rodent tissue as described previously (Kang et al., 1998; Tian et al., 2005). Briefly, tissue taken directly from surgical resection was placed in ice-cold solution containing the following (in mM): 240 sucrose, $2.5 \mathrm{KCl}, 0.5 \mathrm{CaCl}_{2}, 10$ $\mathrm{MgCl}_{2}, 26 \mathrm{NaHCO}_{3}, 1.25 \mathrm{NaH}_{2} \mathrm{PO}_{4}$, and 10 glucose (saturated with $95 \%$ $\mathrm{O}_{2} / 5 \% \mathrm{CO}_{2}$ ). Transverse sections of $400 \mu \mathrm{m}$ were then cut using a vibratome. Slices were then placed in oxygenated artificial CSF (ACSF) (in mм: $125 \mathrm{NaCl}, 2.5 \mathrm{KCl}, 2 \mathrm{CaCl}_{2}, 1 \mathrm{MgCl}_{2}, 26 \mathrm{NaHCO}_{3}, 1.25 \mathrm{NaH}_{2} \mathrm{PO}_{4}$, and 10 glucose) were allowed to recover for $>1 \mathrm{~h}$. Slices were loaded with the calcium indicator fluo- $4 \mathrm{AM}$ (Invitrogen) at $30-32^{\circ} \mathrm{C}$ for $60-80 \mathrm{~min}$ in ACSF containing fluo-4 AM $(10 \mu \mathrm{M})$ and $0.02-0.04 \%$ pluronic $\mathrm{F}-127$, in the presence or absence of $o$-nitrophenyl (NP)-EGTA AM $(200 \mu \mathrm{M})$ (Invitrogen). The slices were then mounted in a perfusion chamber and were viewed using a custom-built laser-scanning microscope (BX61WI, FV300; Olympus) attached to Mai Tai laser (SpectraPhysics). Photolysis was performed using a custom-built system that released an ultraviolet pulse $3 \mu \mathrm{m}$ in diameter as 10 trains [three pulses with a duration of $10 \mathrm{~ms}$ and an interval of $10-30 \mathrm{~ms} ; 30-40 \mathrm{~mW} ; 355 \mathrm{~nm}$, (DPSS Lasers)]. Calcium analysis was performed by quantifying the number of cells that increased florescence $>2$ SDs above baseline florescence after application of drug or photolysis. Drugs used included ATP (100 $\mu \mathrm{M}-5 \mathrm{mM})$, glutamate (1-15 mM), ( \pm )-1-aminocyclopentane-trans-1,3-dicarboxylic acid (t-ACPD) $(30 \mu \mathrm{M})$, and TTX $(1 \mu \mathrm{M})$ and were all purchased from SigmaAldrich. Wave velocity was calculated as the distance from the target divided by the time-to-peak increase in florescence after uncaging and was averaged for all cells within one wave.

\section{Results}

\section{At least four morphologic classes of GFAP-positive cells are present in the human cortex}

At least four major morphologic subclasses of GFAPimmunoreactive cells were identified in the adult human temporal lobe (Fig. 1). Layer 1 is characterized by densely packed interlaminar astrocytes, which extend millimeter long processes terminating in layers $2-4$. Layers $2-6$ are characterized by protoplasmic astrocytes of a larger size than found in rodents. In layers $5-6$, a small number of varicose projection astrocytes reside. These cells extend long processes in a seemingly random manner. Finally, fibrous astrocytes populate the white matter. Similarly, examination of the chimpanzee cortex revealed four subtypes of $\mathrm{GFAP}^{+}$cells (supplemental Fig. 1, available at www.jneurosci. org as supplemental material), although the cellular complexity did not compare with those of human brain. In contrast, cortex of lower-order primates, including the rhesus macaque and squirrel monkeys, contained three subtypes of astrocytes, whereas only protoplasmic and fibrous astrocytes were identified in rodents (supplemental Fig. 1, available at www.jneurosci.org as supplemental material).

\section{Varicose projection astrocytes: a novel GFAP-positive cell in higher-order primate cortex}

One of the most striking features distinguishing humans and chimpanzee from other lower primate and rodent astrocytes was the presence of a previously undescribed pool of morpho- 
logically distinct $\mathrm{GFAP}^{+}$cells residing in layers 5-6, characterized by long fibers with prominent varicosities (Fig. $2 \mathrm{~A}$ ). The main $\mathrm{GFAP}^{+}$processes of these varicose projection astrocytes are straighter than those of protoplasmic astrocytes, whose primary processes are typically tortuous and highly branched (compare with Fig. $4 \mathrm{~B}$ ). Additionally, these cells also extend one to five long processes of up to $1 \mathrm{~mm}$ in length, which may terminate in the neuropil or on the vasculature (Fig. 2A). The processes of these projection astrocytes are characterized by varicosities that are spaced approximately every $10 \mu \mathrm{m}$ (Fig. 2A,C) (see Fig. $3 D$ ). The processes frequently penetrate the process-delimited domains of neighboring astrocytes. These varicose projection astrocytes were relatively sparse but were present in all 30 specimens examined. In our analysis of primate tissue, we were able to locate a small number of varicose projection astrocytes within layers 5 or 6 of the chimpanzee cortex (Fig. $2 A$, inset). These cells differed from those seen in human in that they were smaller and less complex, with fewer main $\mathrm{GFAP}^{+}$processes. However, we believe that they are the same cell type in that they were located in the same cortical loci and laminae, and similarly extended one to three long processes characterized by evenly spaced varicosities. We were unable to perform morphometric analysis to compare these chimpanzee cells directly with their counterparts, given the paucity of higher-order primate tissue available. In other species of primates, including rhesus macaque and squirrel monkeys, we were unable to identify this cell type, suggesting that varicose projection astrocytes are specific to higher-order primates and humans (supplemental Fig. 1, available at www.jneurosci.org as supplemental material). To our knowledge, astrocytes with this morphological phenotype have not been reported in any other species, and the function of their long processes is not known.

GFAP immunolabeling reveals only the filamentous skeleton of an astrocyte.

To visualize the full structure of varicose projection astrocytes, we therefore used DiI-mediated diolistic labeling (biolistic insertion of DiI into cells) of lightly fixed human tissue to better visualize the full extent of single astroglia (Fig. $2 B-F$ ). DiItagged varicose projection astrocytes resemble protoplasmic astrocytes, with one to five long thin processes extending from a relatively symmetrical cell (Fig. $2 B-F$ ). The fine processes of these cells are, however, spiny and sharp, unlike the bulbous processes described previously in rodent protoplasmic astrocytes (Bushong et al., 2002; Ogata and Kosaka, 2002; Ober-
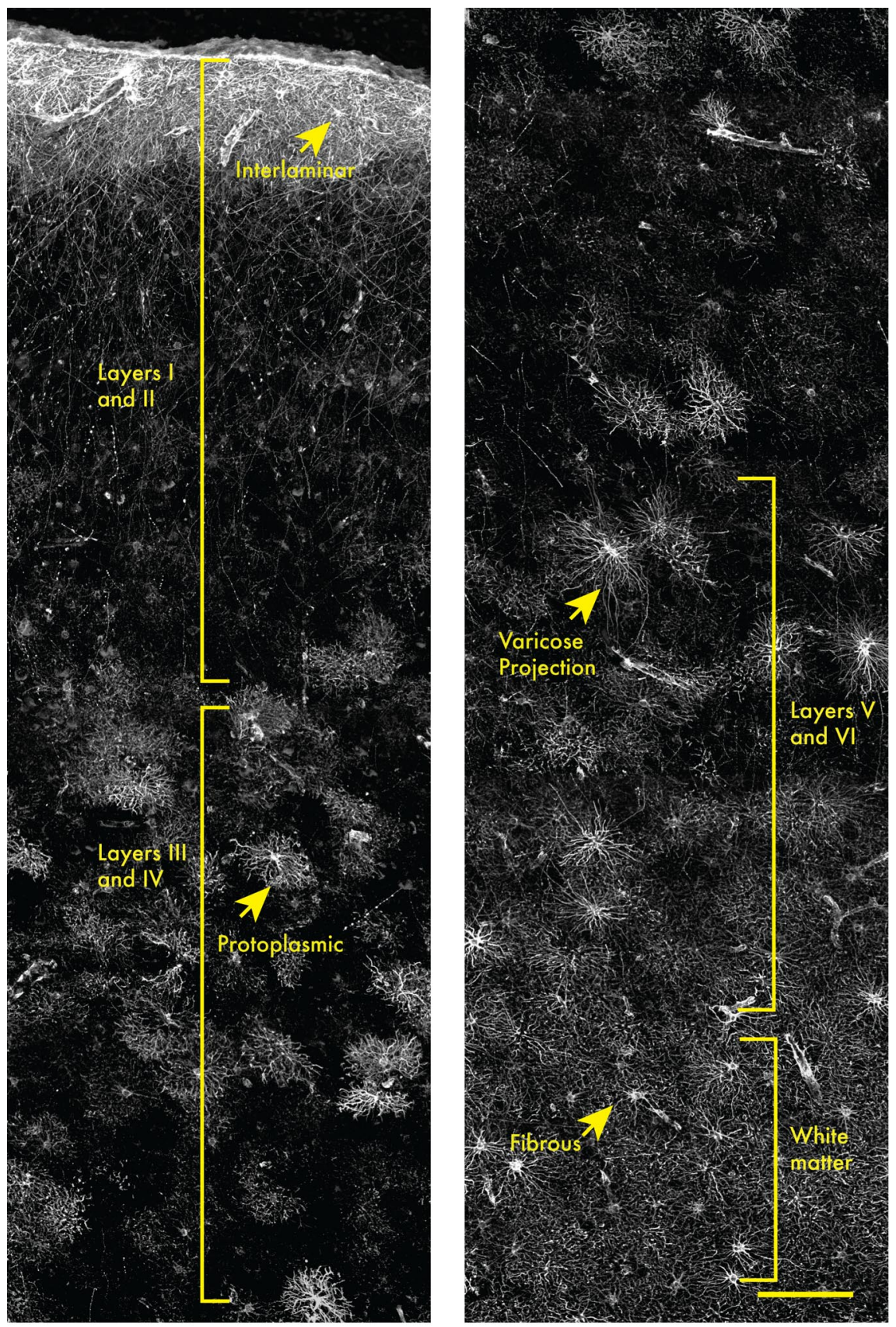

Figure 1. There are four classes of GFAP ${ }^{+}$cells in the human cortex. Human brains were immunolabeled with GFAP and analyzed throughout all layers of the cortex to determine subclasses of human astrocytes. Layer 1 is composed of the cell bodies of interlaminar astrocytes, whose processes extend over millimeter lengths through layers 2-4 and are characterized by their tortuous morphology. Protoplasmic astrocytes, the most common, reside in layers $2-6$. Polarized astrocytes are found only in humans and are seen sparsely in layers $5-6$. They extend millimeter long processes that are characterized by varicosities. Fibrous astrocytes are found in the white matter and contain numerous overlapping processes. Yellow lines indicate areas in which the different classes of astrocytes reside. Scale bar, $150 \mu \mathrm{m}$.

heim et al., 2008) (see Fig. $4 F$ ). Many of the processes from varicose projection astrocytes contacted the vasculature, either through the direct extension of long processes to vascular walls or by en passant contact of glial processes with the capillary microvasculature (Fig. $2 F$ ).

\section{Primate-specific interlaminar fibers in the human cortex}

The GFAP-labeled human cortex exhibited an abundance of interlaminar astrocytes (Fig. 3A-C,E). Interlaminar astrocytes are primate-specific cells that are densely packed and reside solely in 

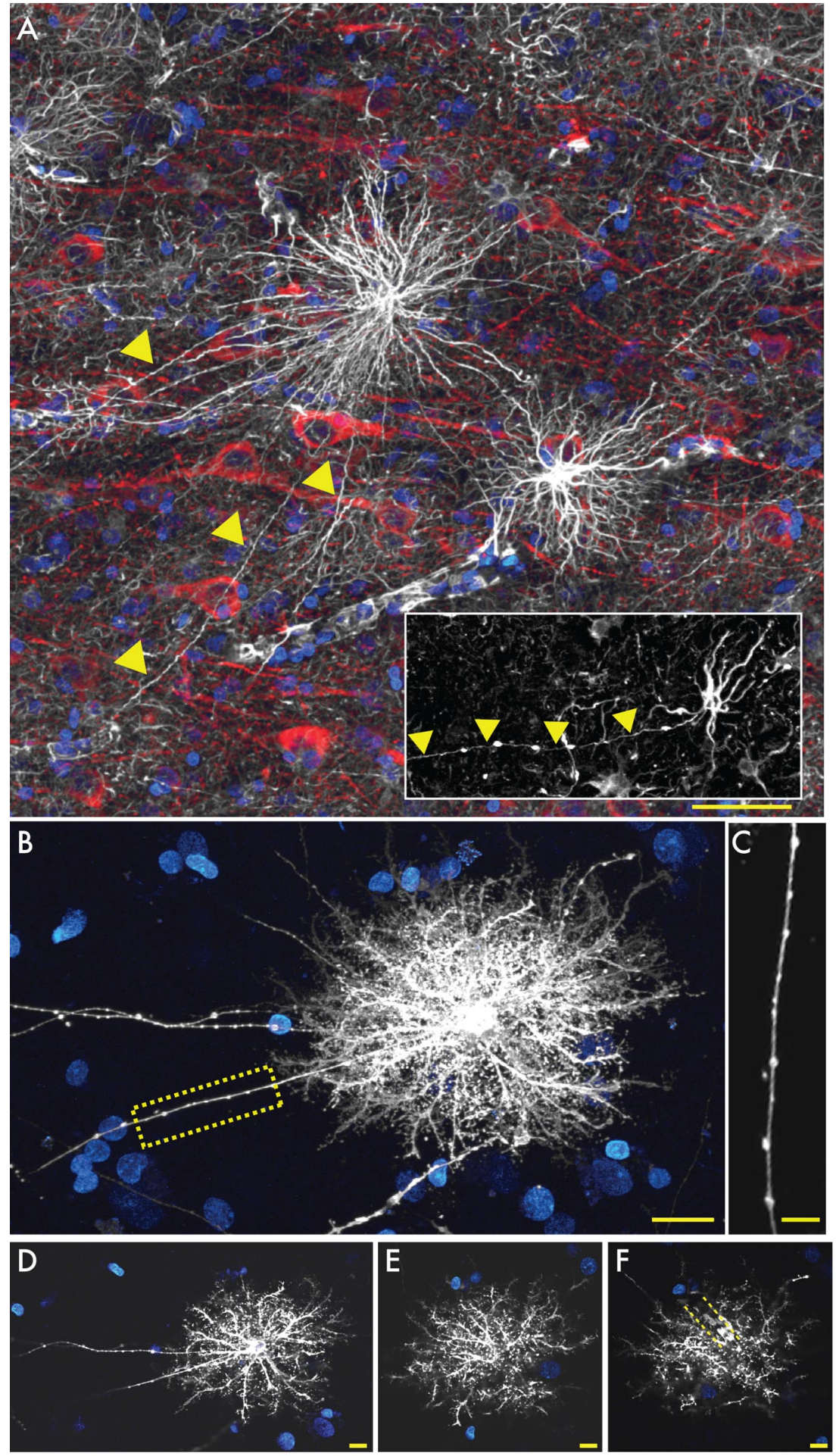

Figure 2. Varicose projection astrocytes in layers 5 and 6 . $A$, Varicose projection astrocytes reside in layers $5-6$ and extend long processes characterized by evenly spaced varicosities. Inset, Varicose projection astrocyte from chimpanzee cortex. GFAP, White; MAP2, red; and DAPI, blue. Yellow arrowheads indicate varicose projections. Scale bar, $50 \mu \mathrm{m}$. $\boldsymbol{B}$, Diolistic labeling (white) of a varicose projection astrocyte whose long process terminates in the neuropil. sytox, Blue. Scale bar, $20 \mu \mathrm{m}$. C, High-power image of the yellow box in $\boldsymbol{B}$ highlighting the varicosities seen along the processes. Scale bar, $10 \mu \mathrm{m}$. $\boldsymbol{D}-\boldsymbol{F}$, Individual $z$-sections of the astrocyte in $\boldsymbol{B}$ demonstrating long processes, straighter fine processes, and association with the vasculature. Yellow dotted lines in $\boldsymbol{F}$ outline position of a blood vessel.

layer 1. They have oblong cell bodies and extend processes both to the pial surface and through the cortical layers in a columnar manner (supplemental Fig. 1, available at www.jneurosci.org as supplemental material). In addition to being more numerous than their chimpanzee counterparts, the morphology of interlaminar astrocytes is subtly different in humans. Human interlaminar astrocytes have small spheroid cell bodies and several short processes that contribute to the pial glial limitains, creating a thick network of GFAP fibers not seen in the primate (Fig. $3 B$, compare with supplemental Fig. 1, available at www. jneurosci.org as supplemental material). These cells also extend one to two processes from layer 1, terminating in layers $2-4$ of the cortex. This results in numerous millimeter-long processes radiating though the outer cortical layers (Fig. 3A). Interlaminar astrocyte processes are tortuous, often manifesting a spiriform or corkscrew pattern of fiber transit (Fig. 3C). The diameter of their processes is constant without varicosities and can therefore easily be distinguished from those of polarized astrocytes (Fig. 3C,D). The processes of interlaminar astrocytes do not respect the borders of protoplasmic astrocytes and frequently travel through their fiber domains (Fig. 3A). The processes terminate in the neuropil and occasionally on the vasculature (data not shown and Colombo and Reisin, 2004). GFAP immunoelectron microscopy showed that these processes frequently contained mitochondria (Fig. 3E) (Colombo et al., 1997). The function of interlaminar astrocytes and their long processes remains unknown.

\section{Human protoplasmic astrocytes are more complex than the rodent}

Protoplasmic astrocytes are the most abundant cell population of the mammalian cortex. However, human protoplasmic astrocytes are both more complex and larger than their rodent and primate counterparts (Fig. $4 A, B$ ) (supplemental Fig. 1, available at www.jneurosci.org as supplemental material). Protoplasmic astrocytes reside in layers 2-6 and vary in domain diameter from $<100$ to $>400 \mu \mathrm{m}$, with an average diameter of $142.6 \pm 5.8 \mu \mathrm{m}$ based on GFAP immunolabeling ( $n=50$ cells from 6 patients). As such, they are 2.55-fold larger than rodent protoplasmic astrocytes, which have an average diameter of $56.0 \pm 2.0 \mu \mathrm{m}(n=65$ cells from 7 animals; $p<0.0001$ ) (Fig. $4 C$ ) (Oberheim et al., 2008). Human protoplasmic astrocytes exhibited an $\sim 2$.6-fold larger length of their longest process, with an average of $97.9 \pm 5.2 \mu \mathrm{m}$ in humans and $37.2 \pm 2.0$ $\mu \mathrm{m}$ in rodents (human, $n=50$ cells from 6 patients; mouse, $n=65$ cells from 7 animals; $p<0.0001$ ) (Oberheim et al., 2006). The diameters of their thickest $\mathrm{GFAP}^{+}$processes also differ (human, $2.9 \pm 0.18 \mu \mathrm{m}$; mouse, $2.2 \pm$ $0.13 \mu \mathrm{m} ; p<0.005$ ) (Oberheim et al., 2008). Most significantly, human astrocytes extended 10 -fold more $\mathrm{GFAP}^{+}$processes from 

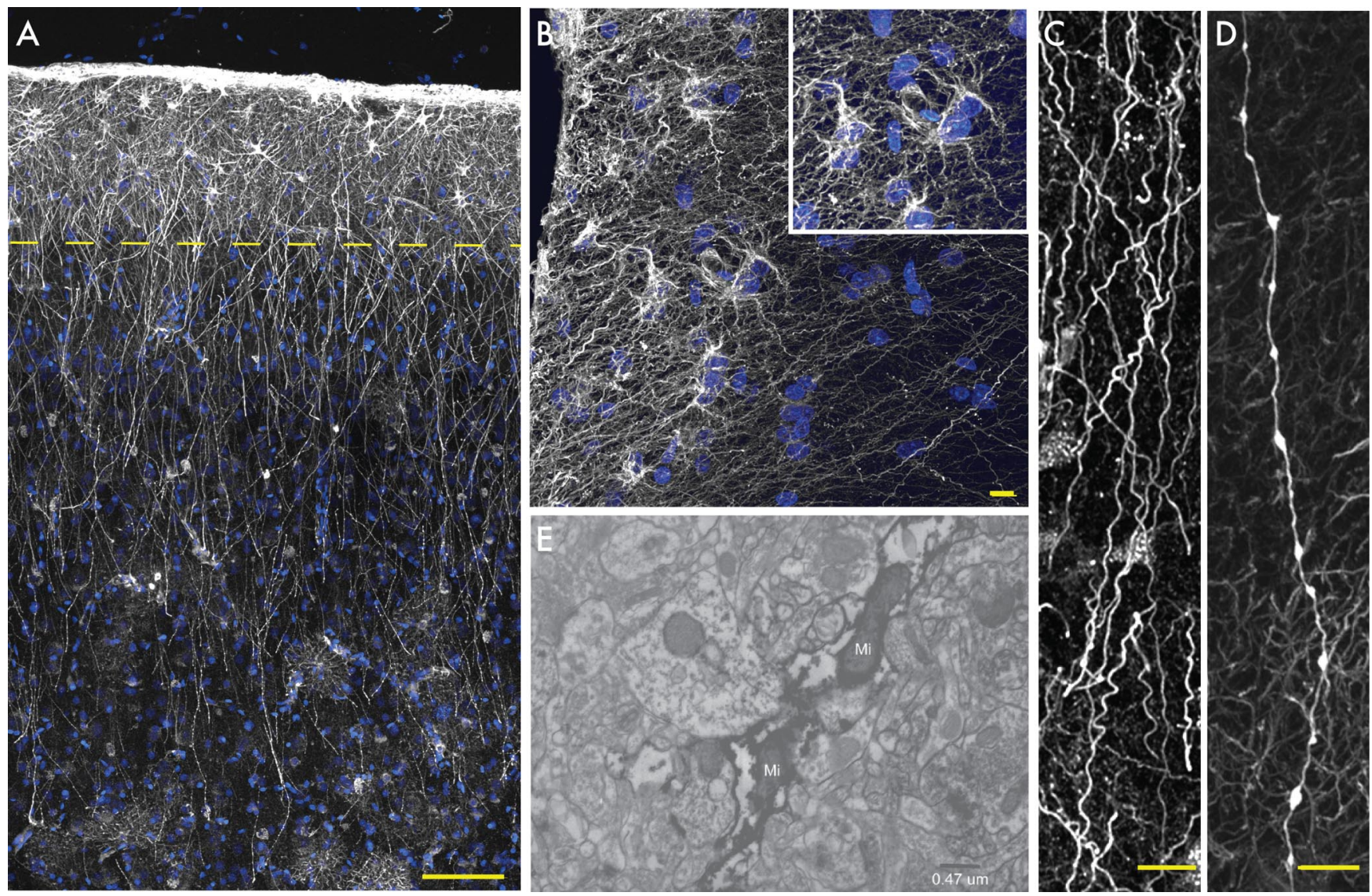

Figure 3. Primate-specific interlaminar astrocytes populate layer 1. A, Pial surface and layers 1-2 of human cortex. GFAP, White; DAPI, blue. Scale bar, $100 \mu \mathrm{m}$. Yellow line indicates border between layer 1 and 2. B, High-power image of layer 1 showing interlaminar astrocytes. Inset, Cell bodies. GFAP, White; DAPI, blue. Scale bar, $10 \mu \mathrm{m}$. C, Interlaminar astrocyte processes characterized by their tortuosity. Scale bar, $10 \mu \mathrm{m}$. D, Process from a varicose projection astrocyte. Scale bar, $10 \mu \mathrm{m}$. $\boldsymbol{E}$, Electron micrograph of GFAP immunohistochemistry demonstrates the presence of mitochondria (Mi) within interlaminar fibers.

their cell body than do rodent astrocytes, with an average of $37.5 \pm$ 5.17 processes radiating from the nucleus compared with $3.75 \pm$ 0.115 in the rodent (human, $n=50$ cells from 7 patients; rodent, $n=$ 65 cells 7 animals; $p<0.0001$ ) (Fig. 4D) (Oberheim et al., 2008).

Protoplasmic astrocytes in nonhuman primates manifest an intermediate phenotype between rodents and humans: they are smaller and less complex than human astrocytes but larger and more complicated that those seen in rodents (supplemental Fig. 1, available at www.jneurosci.org as supplemental material). For example, the average diameter of protoplasmic cortical astrocytes in the chimpanzee brain was $81.7 \pm 1.9 \mu \mathrm{m}(n=36)$, which is significantly smaller than human astrocytes, but significantly larger than protoplasmic astrocytes in mouse brain $(p<0.001$, ANOVA with Bonferroni's post hoc test).

We diolistically labeled both human and rodent tissue with DiI to examine the full structure of the protoplasmic astrocytes (Fig. $4 E, F$ ). The human protoplasmic astrocytes were easily identified based on their characteristic bushy morphology. Diolistic labeling permitted us to discern the numerous delicate GFAP-immunonegative processes of both the rodent and human brain. The fine processes in human protoplasmic astrocytes are bulbous, similar to those described previously in rodent, yet distinctly different from the spiny and sharp processes of varicose projection astrocytes (Fig. $2 B-F$ ). Overall, diolistic labeling revealed the specific morphologic complexity of the human protoplasmic astrocytes.

\section{Human protoplasmic astrocytes are organized in domains}

Rodent protoplasmic astrocytes are organized into domains (Bushong et al., 2002; Ogata and Kosaka, 2002; Halassa et al., 2007). Each astrocyte occupies its own anatomical space with little overlap between adjacent cells. Thus, all neuronal cell bodies, synapses, and vessels within an astrocytic domain will be in contact with processes from a single astrocyte only. It has been speculated that astrocytic domains constitute a unit for local integration and modulation of neural activity that is spatially and temporally distinct from synaptic transmission. To determine whether domain organization has been preserved through evolution and exists in the human cortex, we used diolistic labeling to tag human and rodent cells in situ, with two spectrally distinct lipophilic dyes, DiI and DiD (Fig. $5 A, B)$. When two adjacent astrocytes are labeled with different dyes, the borders between the two cells may be clearly visualized, and their domain organizations can be examined. We found that, like rodent astrocytes, human protoplasmic astrocytes were also organized into domains, with limited overlap between adjacent cells (Fig. $5 B, I-L$ ); the extent of this overlap was quantified in two ways. First, the border of the domain of the cell was outlined by connecting the tips of the processes of each cell. The area common between the two cells was then averaged from 10 optical sections, sampled $1 \mu \mathrm{m}$ apart (Fig. 5C, $F, I$ ). In addition, processes that crossed into the domain of its neighbor were also measured and averaged over 


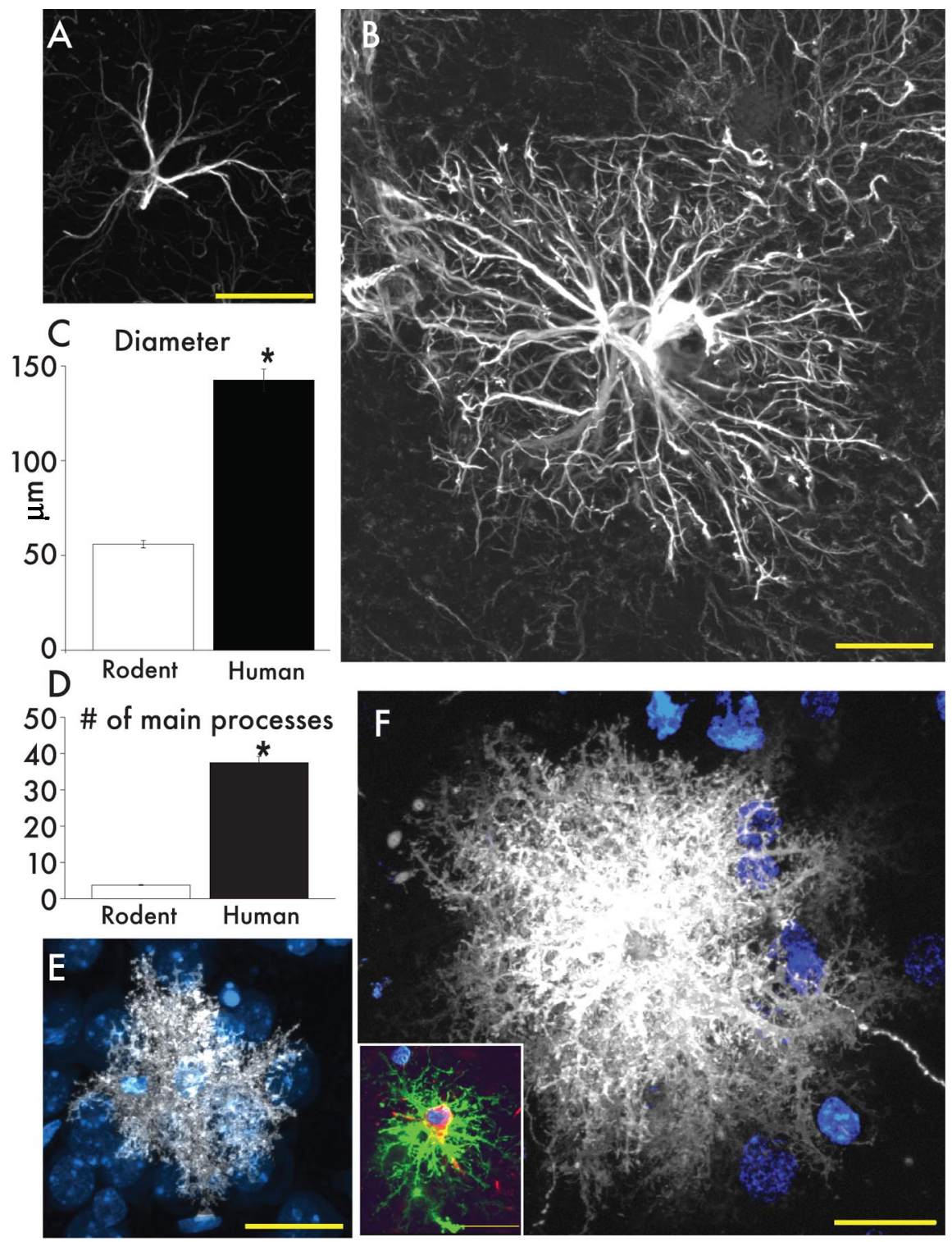

Figure 4. Protoplasmic astrocytes are larger and more complicated than the rodent counterpart. $\boldsymbol{A}$, Typical mouse protoplasmic astrocyte. GFAP, White. Scale bar, $20 \mu \mathrm{m}$. $\boldsymbol{B}$, Typical human protoplasmic astrocyte in the same scale. Scale bar, $20 \mu \mathrm{m}$. $\boldsymbol{C}, \boldsymbol{D}$, Human protoplasmic astrocytes are 2.55-fold larger and have 10-fold more main GFAP processes than mouse astrocytes (human, $n=50$ cells from 7 patients; mouse, $n=65$ cells from 6 mice; mean \pm SEM; ${ }^{*} p<0.005, t$ test). $\boldsymbol{E}$, Mouse protoplasmic astrocyte diolistically labeled with Dil (white) and sytox (blue) revealing the full structure of the astrocyte including its numerous fine processes. Scale bar, $20 \mu \mathrm{m}$. $\boldsymbol{F}$, Human astrocyte diolistically labeled demonstrates the highly complicated network of fine process that defines the human protoplasmic astrocyte. Scale bar, $20 \mu \mathrm{m}$. Inset, Human protoplasmic astrocyte diolistically labeled as well as immunolabeled for GFAP (green) demonstrating colocalization. Scale bar, $20 \mu \mathrm{m}$.

10 optical sections (Fig. $5 D, G, H, K, L$ ). We predicted that human domains would exhibit more overlap than rodent cells because of their significantly greater size. The average area of overlap for each human astrocytic domain was $204.7 \pm 44.1$ $\mu \mathrm{m}^{2}$, whereas the summated length of overlapping processes was $53.0 \pm 10.7 \mu \mathrm{m}(n=10$ cells from 2 patients $)$. As predicated, this was significantly greater than in corresponding rodent cortical samples, in which the average area of overlap was $11.8 \pm 2.2 \mu \mathrm{m}^{2}$, comprising processes with net overlapping length of $4.2 \pm 0.7 \mu \mathrm{m}(n=30$ cells from 7 animals; area comparison significant to $p<0.0001$; overlapping process lengths to $p<0.0001$ ) (Oberheim et al., 2008). The area of overlap in humans was larger than the predicted 6.5-fold increase, based on the 2.55 -fold increase in diameter. Thus, hu- man protoplasmic astrocytes were distributed evenly across cortex and were organized in domains, albeit with more extensive overlap of their processes than in rodents.

\section{Human protoplasmic astrocytes have} increased GFAP in their end feet

End feet are specialized structures of protoplasmic astrocytes that contact the vasculature and contribute to the bloodbrain barrier (Simard and Nedergaard, 2004). Astrocytic end feet can be seen as distinct compartments with polarized expression of a number of proteins, such as aquaporin 4, $\mathrm{Cx} 43$, and Kir4.1 (Nagelhus et al., 2004; Simard and Nedergaard, 2004). To compare the end feet of human and rodent astrocytes, we prepared acute slices identically from both species and immunolabeled with GFAP (Fig. 6A-E). In both species, nearly every protoplasmic astrocyte contacted the vasculature, either through extension of long processes or capillaries within its domain. However, the microanatomy of their respective end feet manifestly differed. In the human brain, GFAP completely encompassed the vessels, creating a cobblestone pattern along the vasculature (Fig. $6 C-E$ ). In contrast, rodent astrocytes formed rosettes of GFAP around the vasculature (Fig. $6 A, B$ ). Ultrastructurally, the astrocytic end feet of humans and rats appeared similar; each contained an abundance of mitochondria (Fig. $6 F$, arrows).

\section{Human fibrous astrocytes are} significantly larger than rodent Fibrous astrocytes, found in the white matter of both rodents, primates, and humans, are morphologically distinct from protoplasmic astrocytes. In rodents, they are larger than protoplasmic astrocytes, with an average GFAP-defined domain diameter of $85.6 \pm 2.7 \mu \mathrm{m}(n=50$ cells from 3 mice; $p<0.0001$ ) (Fig. $7 A, C)$. Their processes are radially oriented in the direction of the axon bundles. When viewed after DiI-based diolistic labeling, they were noted to have fewer fine processes, resulting in a much smaller surface area-to-volume ratio than protoplasmic astrocytes (Fig. 7E, compare with Fig. 4F). Human fibrous astrocytes proved significantly larger than their rodent counterparts, with an average GFAP-defined domain diameter of $183.2 \pm 6.1$ $\mu \mathrm{m}(n=50$ cells from 6 patients; $p<0.0001$ rodent) (Fig. $7 B, C)$. Their relatively unbranched straight GFAP-positive processes extended evenly from a small cell body. These processes interdigitate extensively with processes from neighboring fibrous astrocytes; therefore, fibrous astrocytes are not organized within domains (Fig. 7B). Their cell bodies, however, are equally spaced (Fig. 1). This regular spacing is most likely attributable to the structural support that the fibrous astrocytes provide for the ax- 

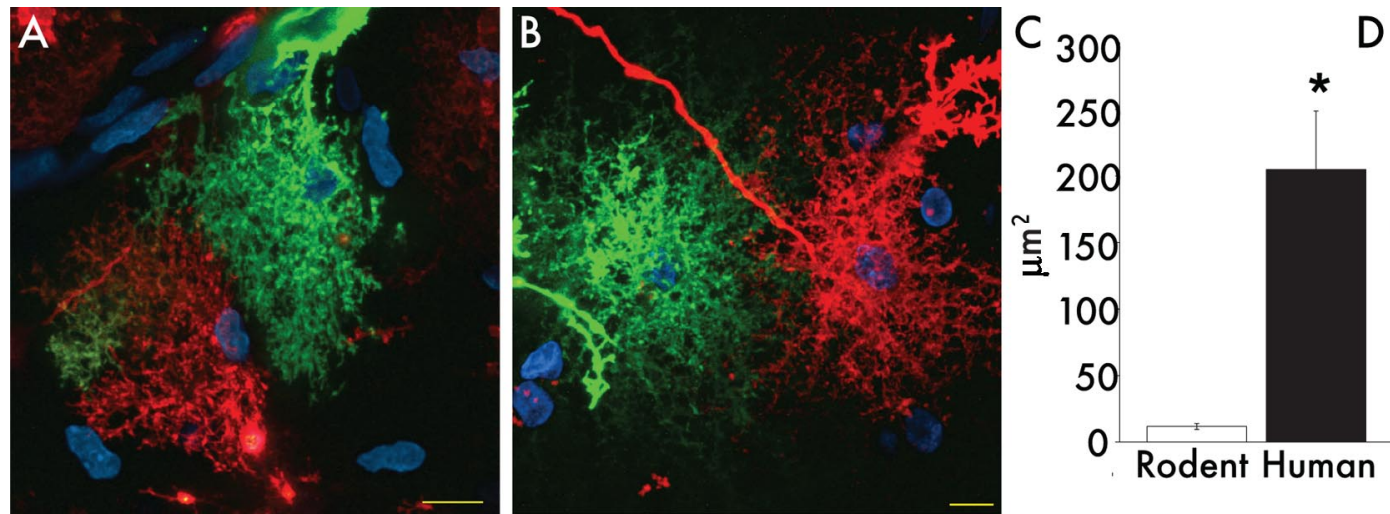

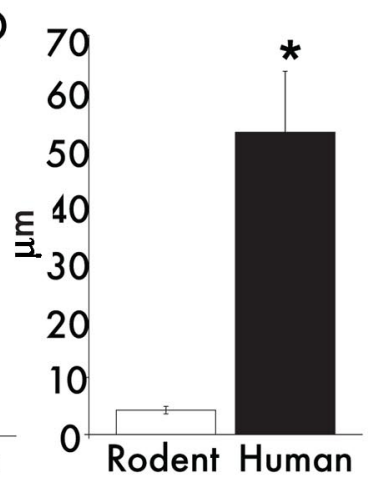

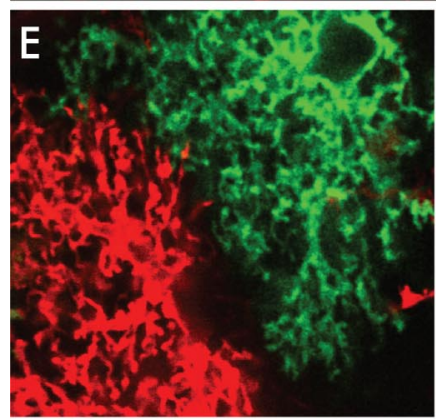
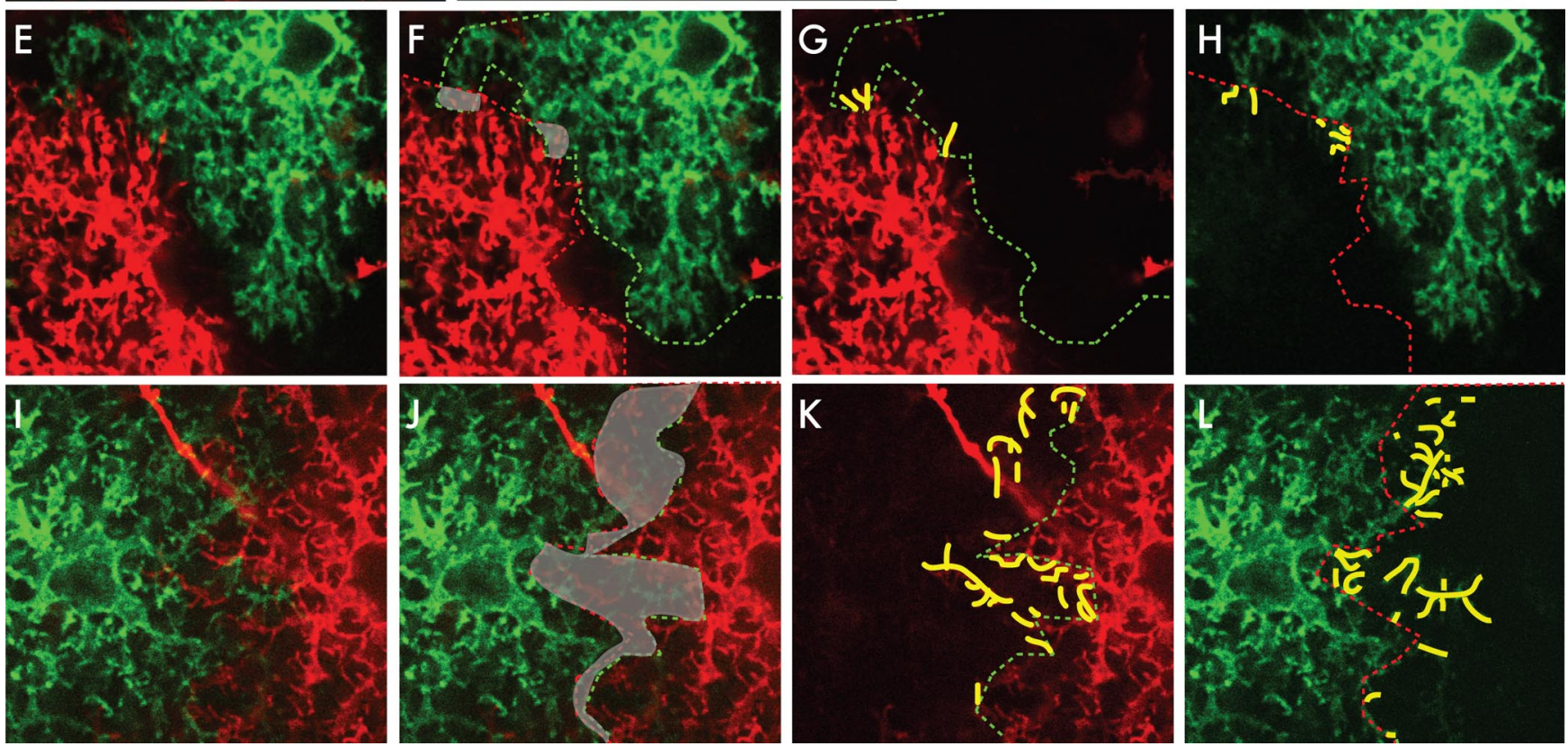

Figure 5. Human astrocytes are organized into domains. $A$, Cortical mouse astrocytes labeled with Dil (red) and DiD (green) demonstrating the presence of domains. Nuclei (sytox), Blue. Scale bar, $10 \mu \mathrm{m} . \boldsymbol{B}$, Human protoplasmic astrocytes labeled with Dil (red) and DiD (green) demonstrating the presence of domains. Scale bar, $10 \mu \mathrm{m}$. C, D, Human astrocytes have greater overlap in their domains than rodents. (human, $n=10$ cells from 2 patients; mouse, $n=30$ cells from 7 mice; mean \pm SEM; ${ }^{*} p<0.005, t$ test). $\boldsymbol{E}-\boldsymbol{H}$, High-power images of domain boundaries between neighboring mouse astrocytes seen in $\boldsymbol{A}$. I $\boldsymbol{L}$, , High-power images of domain boundaries between neighboring human astrocytes seen in $\boldsymbol{B}$. Green lines represent the borders of the green cells, and red lines represent the borders of the red cells. Area of overlap between cells is delineated in gray. Yellow lines indicate processes that transverse over the border of the adjacent cell.

ons tracts. Additionally, the majority of human fibrous astrocytes contact the vasculature, either close to their cell bodies or through the extension of long processes. Diolistic labeling reveals that human fibrous astrocytes also have essentially none of the fine bulbous processes characteristic of the protoplasmic astrocytes found in the human cortex. (Fig. 7D).

\section{Functional properties of human astrocytes}

To study $\mathrm{Ca}^{2+}$ signaling in human astrocytes, we prepared acute slices from human cortex immediately after surgical resection. The tissue was placed in ice-cold oxygenated ACSF, sectioned within 15 min after surgical resection, and then loaded with the calcium indicator fluo-4 AM and, in some cases, with caged $\mathrm{Ca}^{2+}$, NP-EGTA AM. $\mathrm{Ca}^{2+}$ signals were then visualized using a two-photon laser-scanning microscope.

To determine whether human astrocytes were able to support a calcium wave, we photoreleased caged calcium (NP-EGTA) in astrocytes in situ, in the presence or absence of TTX (Fig. $8 B$ ). Photorelease of caged calcium led to an increase in intracellular calcium in the target cell, with subsequent calcium wave propagation to other cells in 25 of 28 experiments in the presence of
TTX (six slices from two patients) and in seven of seven experiments in the absence of TTX (four slices from one patient). The speed of the calcium wave in the absence of TTX was $43.4 \pm 4.7$ $\mu \mathrm{m} / \mathrm{s}$ ( $n=7$ waves); this was not significantly altered by TTX, added so as to block concurrent neuronal activity $(36.3 \pm 4.4$ $\mu \mathrm{m} / \mathrm{s} ; n=23$ waves; $p=0.41$ ).

To compare the rates of calcium wave transmission between human and rodent astrocytes, we next measured the speed of calcium wave transmission in TTX-treated rodent cortical slices prepared identically to their human counterparts. We found that the speed of the calcium wave in rodents was significantly slower than in humans: mouse astrocytes propagated calcium waves at $8.6 \pm 0.6 \mu \mathrm{m} / \mathrm{s}$ ( $n=8$ waves from 3 animals), less than a one-fifth the speed of their human counterparts $(43.4 \pm 4.7 \mu \mathrm{m} / \mathrm{s} ; n=7$; $p<0.005$ ) (Fig. 8A)

Adult astrocytes in the rodent brain respond to both ATP and glutamate (Volterra and Meldolesi, 2005). To assess the responses of human astrocytes to these stimuli, we next challenged human astrocytes with bath application of ATP (Fig. 9A). The cells responded to concentrations of ATP ranging from $100 \mu \mathrm{M}$ to $5 \mathrm{~mm}$ by increasing intracellular calcium, in both the presence 

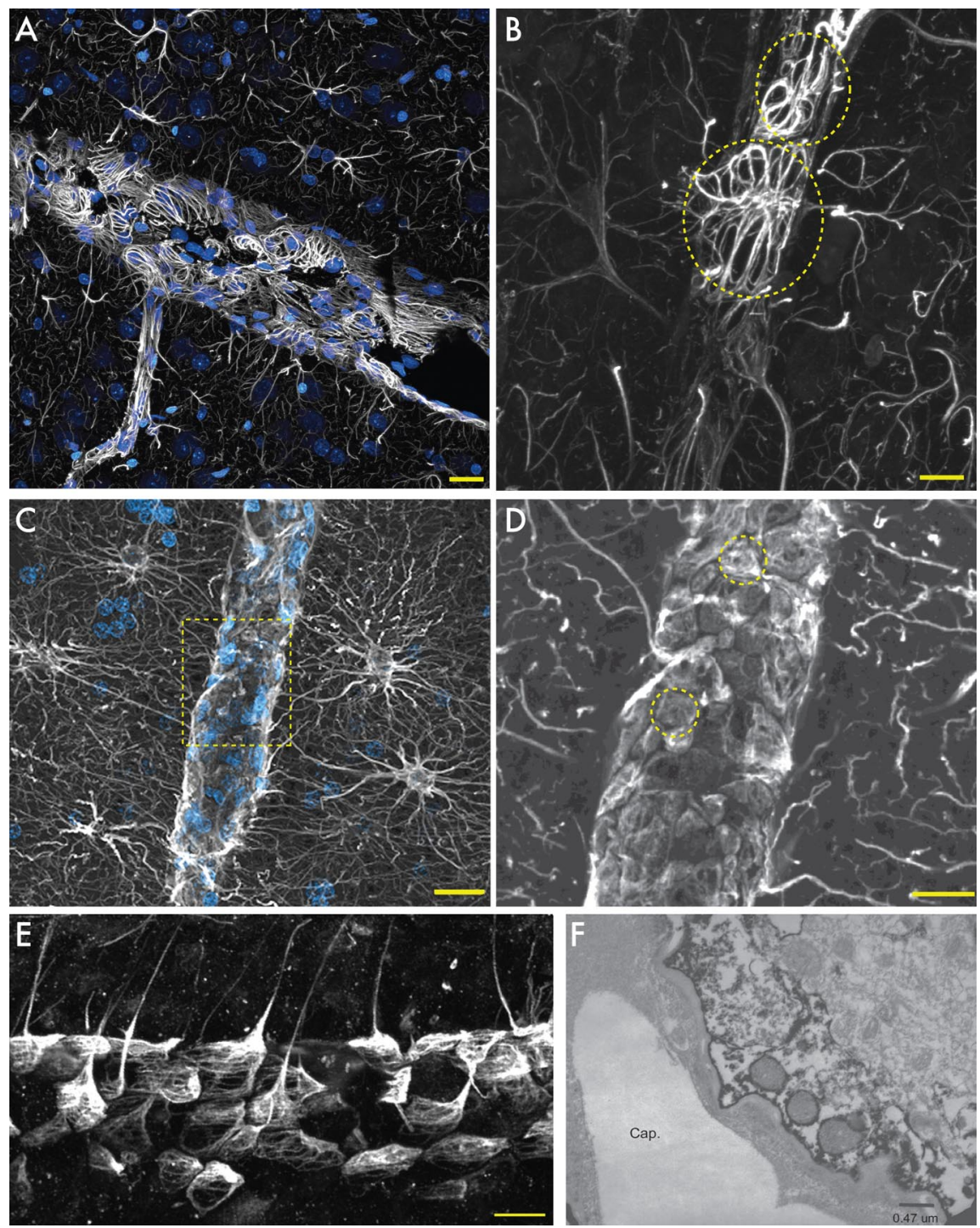

Figure 6. Astrocytic end feet in rodents and humans. $A$, Cortical blood vessel in rat. GFAP, White; nuclei (DAPI), blue. Scale bar, $20 \mu \mathrm{m} . \boldsymbol{B}$, GFAP in end feet forms rosettes on the vessel in the rat. Scale bar, $10 \mu \mathrm{m}$. Yellow circles indicate individual end feet. $\boldsymbol{C}$, Human protoplasmic astrocytes extend processes to the vasculature. Scale bar, $20 \mu \mathrm{m}$. D, Yellow box seen in $\boldsymbol{C}$. GFAP in end feet completely covers the vasculature. Yellow circles indicate individual end feet. Scale bar, $10 \mu \mathrm{m}$. $\boldsymbol{E}$, Transverse section of blood vessel and human astrocyte end feet. Scale bar, $20 \mu \mathrm{m}$. $\boldsymbol{F}$, Electron micrograph of aquaporin 4 immunohistochemistry of the astrocytic end foot on a capillary (Cap.). Note the presence of mitochondria in the end foot of the astrocyte.

and absence of TTX. In the absence of TTX, $93 \pm 3 \%$ of loaded cells responded to ATP by increasing their florescence by $>2$ SDs from their baseline ( $n=161$ cells; 9 slices from 3 patients) (Fig. $9 A$ ). In the presence of TTX, $71 \pm 15 \%$ of cells responded to ATP application (67 cells; 3 slices from 1 patient) (Fig. 9B). In general, astrocytes returned to baseline calcium levels within 20-30 s. In some slices, astrocytes in layer 1 were imaged (Fig. 9A). In addition to cell bodies, processes from these cells also loaded with the calcium indicator dye. Interestingly, in response to ATP stimulus, the processes of these cells also increased their intracellular calcium and displayed propagation of the calcium signal along the interlaminar fibers (Fig. 9A). Thus, human astrocytes respond to ATP through rises in intracellular calcium in both their cell bodies and processes.

Slices were also exposed to glutamate (1-15 mM) by bath application in the presence of TTX (Fig. 9 C). On average, $60 \pm 8 \%$ of the loaded astrocytes responded with rises in intracellular cal- cium ( 54 cells, 4 slices from 3 patients). To determine whether this response to glutamate was mediated by metabotropic glutamate receptors, slices were then exposed to t-ACPD, a group I and II metabotropic glutamate receptor agonist in the presence of TTX (Fig. 9D). A total of $79 \pm 17 \%$ of loaded cells responded to $30 \mu \mathrm{m}$ t-ACPD (64 cells, 2 slices from 1 patient). Astrocytes exhibited a rapid rise in intracellular calcium in response to both glutamate and $\mathrm{t}-\mathrm{ACPD}$ and a return to baseline levels within seconds. Some cells maintained an oscillatory response, with serial elevations in intracellular calcium in response to the initial stimulus. Therefore, human astrocytes, like their rodent counterparts, respond to ATP and glutamate through rises in intracellular calcium. However, the signal propagation by human astrocytes is significantly and substantially more rapid than that exhibited by rodent astroglia.

\section{Discussion}

Classically, astrocytes have been categorized based on morphology. We here compared rodent and primate astrocytes and found that astrocytes in the human brain are characterized by a marked increase in both their microanatomic complexity and in the phenotypic diversity of $\mathrm{GFAP}^{+}$ cells. Human protoplasmic astrocytes are several fold larger and extend 10-fold more $\mathrm{GFAP}^{+}$processes than rodent astrocytes. Although the overlap of processes was somewhat higher in human than rodents, astrocytes were clearly organized in domains in both species.

The existence of interlaminar astrocytes described by Colombo and coworkers was confirmed, and we identified a novel morphologic cell type, varicose projection astrocytes, which were present only in cortical layers $5 / 6$ of higher-order primates. In addition, we found that in acute slice preparation, human astrocytes propagated calcium signals severalfold faster than did rodent astrocytes and responded by marked increases in cytosolic calcium to purinergic and metabotropic glutamate receptors agonists.

\section{Varicose projection astrocytes}

A population of astrocytes bearing varicose projections in layers 5 and 6 was identified and designated as varicose projection astrocytes. To our knowledge, this morphologic cell type has not been described in any infraprimate species. We identified varicose projection astrocytes in humans and chimpanzees but not in lowerorder primates. Varicose projection astrocytes proved relatively sparse but were present in all 30 human samples analyzed. Although this $\mathrm{GFAP}^{+}$cell type was present in chimpanzee brain, it was clearly smaller and less complex than the varicose projection astrocytes residing in human brain.

Varicose projection astrocytes were characterized by shorter, 
more spiny processes than exhibited by typical protoplasmic astrocytes and typically extended one to five essentially unbranched, millimeter long fibers within the deep layers of the cortex. The processes of the varicose projection astrocytes did not respect the domain organization, because they traveled in all directions, piercing and traversing the domains of neighboring protoplasmic astrocytes. Their process morphology was also intriguing; the evenly spaced varicosities suggest specialized structures or compartmentalization of cellular elements along the great distance of the fibers. Because of the rarity of these cells, we were not able to evaluate whether they supported propagation of calcium signals. We hypothesize based on their distinct morphology that these cells are specialized for long-distance communication across cortical layers or even between gray and white matter. Several of their processes terminated on vessels, suggesting that the varicose projections may play a role in coordinating functional hyperemia across more expansive areas than those covered by protoplasmic astrocytes.

\section{Interlaminar astrocytes}

One of the most prominent differences between the brains of primates and other mammals are the interlaminar astrocytes. Although represented in similar numbers in humans and both Old and New World monkeys, interlaminar astrocytes are reduced in both number and length in phylogenetically more distant primates, such as the lemur Microcebus (Colombo et al., 1995, 1997, 1998). Additionally, we have observed that there is a distinct difference in morphology in interlaminar astrocytes in primates and humans. The functional significance of these cells remains unknown (Colombo and Reisin, 2004). Their fibers extend from the pial surface through several layers of cortex before terminating in layers II through IV, yet their tortuosity suggests that they snake through the neuropil, contacting great numbers of other cells along the way. Like the varicose projection astrocytes, the interlaminar fibers do not respect the domain organization and rather penetrate the domains of intervening protoplasmic astrocytes as they traverse the cortex.

In light of the length of interlaminar fibers and the large numbers of cells that each fiber may contact in its cortical path, their capacity to respond to both purinergic and glutaminergic stimulation with calcium elevation, and thence to propagate calcium waves, is of potential importance (Fig. 9A). Of note, the calcium increases in interlaminar cells could be triggered independently in both the cell bodies and fibers. The function of the interlaminar fibers are unknown, but these traits suggest that they may provide a network for the long-distance coordination of intracortical communication thresholds; in addition, because many of their fibers terminate on the capillary microvasculature, interlaminar astrocytes may also play a role in coordinating blood flow among functionally related regions. Interestingly, a progressive loss of the interlaminar fibers have been noted in several disease states associated with multilaminar neuronal loss, including Down's syndrome and Alzheimer's disease (Colombo et al., 2002, 2005). Together, these observations suggest the importance of interlaminar astrocytes to normal cortical function, as well as to the specific cognitive competence of both higher primates and humans.

\section{Protoplasmic astrocytes}

The protoplasmic astrocytes in humans are significantly larger and more complex than the rodent counterpart but are similar to rodents organized in nonoverlapping domains. The increase in size of protoplasmic astrocytes appears to follow the complexity of higher brain functions. The 2.55-fold increase in diameter of human protoplasmic astrocytes corresponds to a 16.5 -fold increase in cellular volume. Synaptic density is relatively preserved throughout evolution with 1397 million synapses $/ \mathrm{mm}^{3}$ in rodents compared with $\sim 1100$ million synapses $/ \mathrm{mm}^{3}$ in the cortex of man (DeFelipe et al., 2002). The volume of astrocytic domains 


\section{A Rodent}
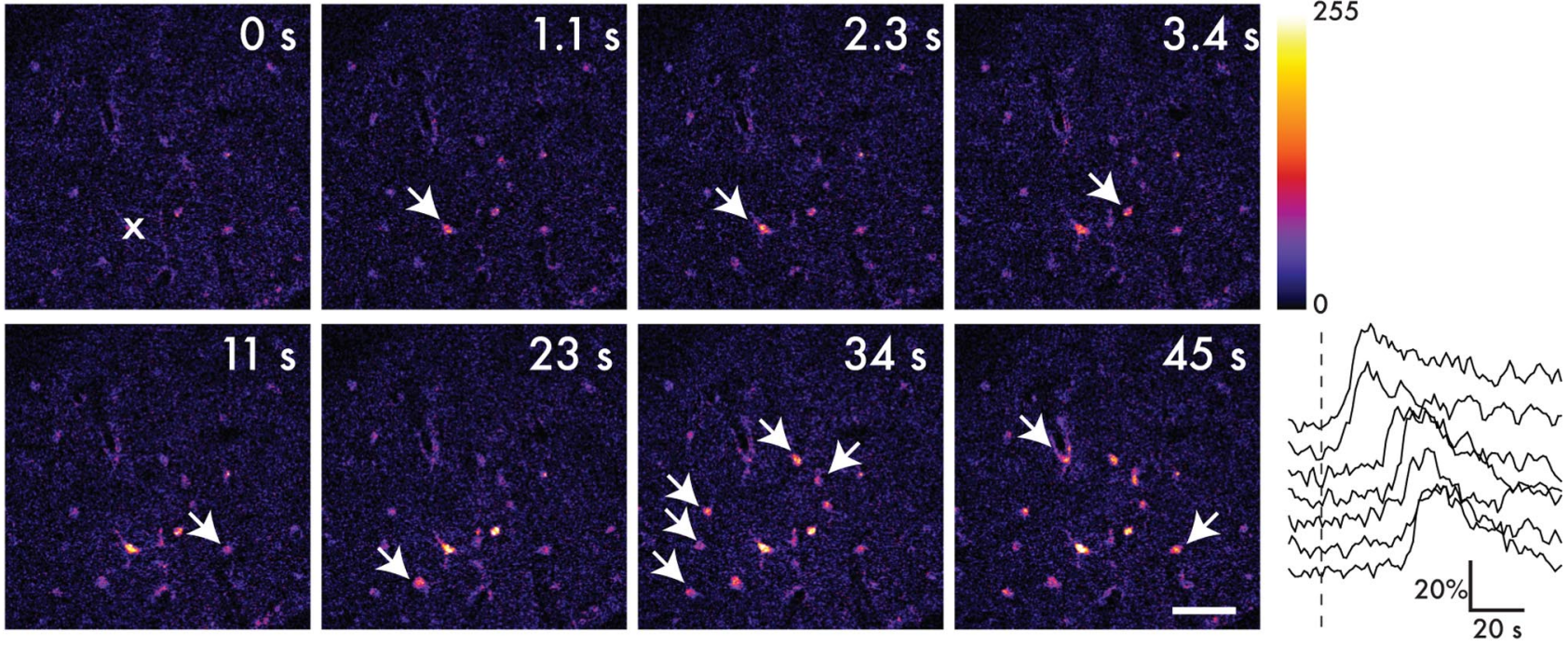

B Human
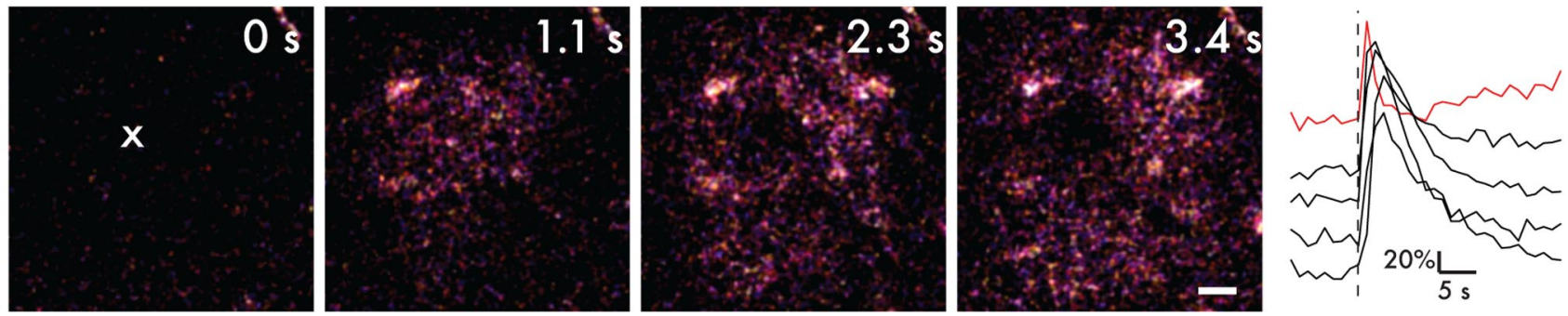

Figure 8. Human astrocytes can support faster calcium waves. $A$, Acute slice preparation of mouse cortex was loaded simultaneously with fluo-4 AM as well as NP-EGTA AM caged calcium. A single astrocyte (indicated as $X$ ) was the target of photorelease of caged calcium in the presence of $1 \mu \mathrm{m}$ TTX. The target cell increased its calcium with uncaging, and, subsequently, other astrocytes in the field also increased their intracellular calcium in a wave manner temporally (white arrows). The graph represents percentage change in fluorescence over time that is indicative of calcium concentrations. As one moves away from the target cell, the time-to-peak of calcium concentration increases, indicating a calcium wave. The target cell as well as other cells in the field returned to baseline calcium levels within seconds. Scale bar, $50 \mu \mathrm{m}$. B. Acute slice preparation of human temporal lobe cortex loaded with fluo-4 AM as well as NP-EGTA AM caged calcium. A single target astrocyte $(X)$ underwent photorelease of caged calcium, resulting in a subsequent calcium wave. The graph represents change in fluorescence over time. Scale bar, $10 \mu \mathrm{m}$.

in rodents has been reported to range from 14,700 and 22,906 $\mu \mathrm{m}^{3}$ in the cortex to 65,900 and $85,300 \mu \mathrm{m}^{3}$ in the hippocampus (Bushong et al., 2002; Ogata and Kosaka, 2002; Chvatal et al., 2007; Halassa et al., 2007). Thus, whereas a domain of a rodent astrocyte covers $\sim 20,000-120,000$ synapses, human astrocytes can cover from $\sim 270,000$ to 2 million synapses within a single domain. If we regard an astrocytic domain as an elementary unit of brain that monitors, integrates, and potentially modifies the activity of a contiguous set of synapses, this glio-neuronal unit in human brain contains far-larger numbers of synapses, thus capable of performing more complex processing per glio-neuronal unit than any other species. It is tantalizing to propose that the computational power of cortex increases as a function of the size of its astrocytic domains. If so, glio-neuronal-based processing may increase the intelligence of primates disproportionately relative to what is accounted for on the basis of brain size alone.

\section{Fibrous astrocytes}

Just as in rodents and nonhuman primates, human fibrous astrocytes are significantly larger than protoplasmic cells. In addition to their larger diameter, they have less fine bulbous processes, consistent with the lack of synaptic contacts in the white matter. These cells seem to be specialized for structural support, in that they have numerous overlapping processes and evenly spaced cells bodies. The increase in size between rodent and human is similar to the ratio seen between protoplasmic astrocytes and therefore may represent a conserved and optimized increase based on enlarged brain size.

\section{Caveats}

This study was restricted to tissues deemed normal by pathological criteria. In most patients, cortex was resected to gain access to deeper located pathologies. Nevertheless, a diseased brain may exhibit more global changes, especially in patients suffering from medication-refractory epilepsy. Notably, however, one of our samples was removed for decompression in the setting of a brain abscess. The fine structure of astrocytes in this patient was similar to the other brains examined. Thus, the analysis presented in this report describes the morphology and organization of astrocytes in normal human brain. We found in initial experiments that the delicate cellular details of astrocytes were lost in autopsy material and decided therefore to only include surgical specimens in the study.

Previous studies on human astrocytes in sections prepared from surgical resected material have analyzed their electrophysiological properties. The limited tissue included in the studies has made it difficult to draw conclusions on the differences between humans and rodents. Bordey and Sontheimer (1998) have shown there is no significant difference between the mean resting potential of human astrocytes compared with rat hippocampal astro- 
cytes. The membrane capacitance, however, is significantly larger in human astrocytes, consistent with the larger cell size (Bordey and Sontheimer, 1998). To our knowledge, this study includes the first demonstration of the functional calcium imaging of human astrocytes in situ. We show that human astrocytes, similar to those of rodents, respond to ATP and glutamate by increasing intracellular calcium and that the long processes of the interlaminar astrocytes supported propagation of calcium waves.

Importantly, we noted that signal propagation by human astrocytes proceeded at much higher velocities among human astrocytes relative to rodent astroglia. In general, human astrocytes propagated calcium waves at a speed more than fourfold faster than in mice. The faster wave propagation of human astroglia may be a consequence of adaptive evolutionary changes, intended to support rapid communication over greater distances. It parallels the increased diameter, myelination, and longer axons that consequently lead to faster propagation velocity of action potentials in humans (Harrison et al., 2002; Wang et al., 2008).

This study is the first to highlight the morphological and functional distinctions between human astrocytes and those of experimental animals. Our observations point out the limitations of using rodent models to study human glial biology and draw attention to the unique features of human astrocytes. We posit that these uniquely human aspects of astrocytic morphology and physiology may provide the cellular substrate for many of the distinct neurological capabilities that make us human.

\section{References}

Andriezen WL (1893) The neuroglia elements of the brain. BMJ 2:227-230.

Benediktsson AM, Schachtele SJ, Green SH, Dailey ME (2005) Ballistic labeling and dynamic imaging of astrocytes in organotypic hippocampal slice cultures. J Neurosci Methods 141:41-53.

Bordey A, Sontheimer H (1998) Properties of human glial cells associated with epileptic seizure foci. Epilepsy Res 32:286-303.

Bushong EA, Martone ME, Jones YZ, Ellisman MH (2002) Protoplasmic astrocytes in CA1 stratum radiatum occupy separate anatomical domains. J Neurosci 22:183-192.

Chvátal A, Anderová M, Kirchhoff F (2007) Three-dimensional confocal morphometry: a new approach for studying dynamic changes in cell morphology in brain slices. J Anat 210:671-683.

Colombo JA, Reisin HD (2004) Interlaminar astroglia of the cerebral cortex: a marker of the primate brain. Brain Res 1006:126-131.

Colombo JA, Yáñez A, Puissant V, Lipina S (1995) Long, interlaminar astroglial cell processes in the cortex of adult monkeys. J Neurosci Res 40:551-556.

Colombo JA, Gayol S, Yañez A, Marco P (1997) Immunocytochemical and electron microscope observations on astroglial interlaminar processes in the primate neocortex. J Neurosci Res 48:352-357.

Colombo JA, Härtig W, Lipina S, Bons N (1998) Astroglial interlaminar processes in the cerebral cortex of prosimians and Old World monkeys. Anat Embryol (Berl) 197:369-376.
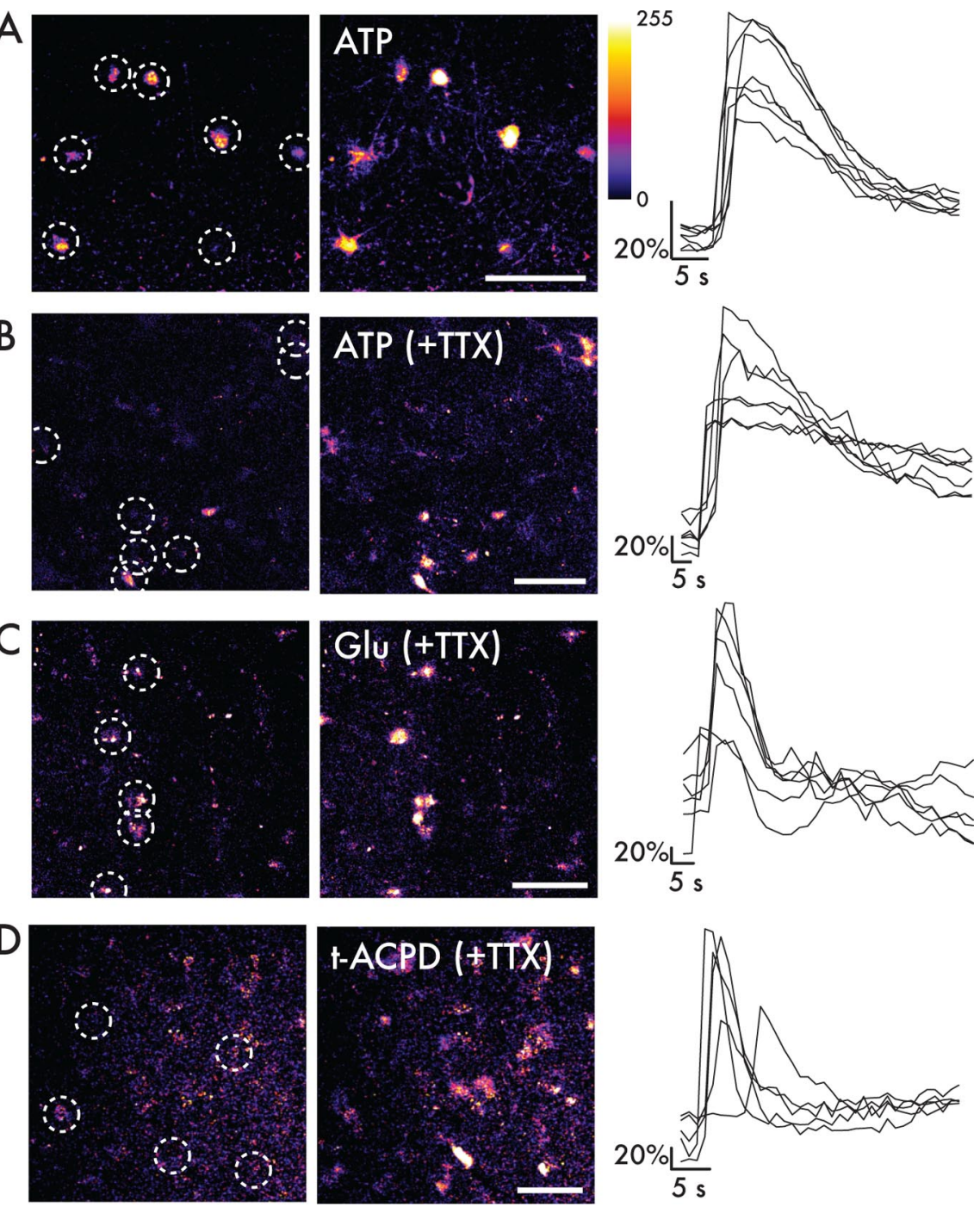

Figure 9. Human astrocytes in situ have functional purinergic and glutamatergic receptors and respond with increases in intracellular calcium. A, Acute slice preparation of human temporal lobe loaded with calcium indicator dye fluo-4 AM. Astrocytes (white circles) were shown with resting $\mathrm{Ca}^{2+}$ levels. After $100 \mu \mathrm{m}$ ATP by bath application, astrocytes increased their intracellular

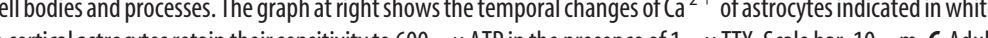
application. Astrocytes respond by increasing intracellular calcium and return to baseline calcium levels within seconds. $\boldsymbol{D}$, Astrocytes respond to bath application of $30 \mu \mathrm{m}$ t-ACPD in the presence of $1 \mu \mathrm{m}$ TTX. Scale bar, $10 \mu \mathrm{m}$.

Colombo JA, Quinn B, Puissant V (2002) Disruption of astroglial interlaminar processes in Alzheimer's disease. Brain Res Bull 58:235-242.

Colombo JA, Reisin HD, Jones M, Bentham C (2005) Development of interlaminar astroglial processes in the cerebral cortex of control and Down's syndrome human cases. Exp Neurol 193:207-217.

DeFelipe J, Alonso-Nanclares L, Arellano JI (2002) Microstructure of the neocortex: comparative aspects. J Neurocytol 31:299-316.

Gan WB, Grutzendler J, Wong WT, Wong RO, Lichtman JW (2000) Multicolor "DiOlistic" labeling of the nervous system using lipophilic dye combinations. Neuron 27:219-225.

Goldman SA, Nedergaard M (1992) Newly generated neurons of the adult songbird brain become functionally active in long-term culture. Brain Res Dev Brain Res 68:217-223.

Halassa MM, Fellin T, Takano H, Dong JH, Haydon PG (2007) Synaptic islands defined by the territory of a single astrocyte. J Neurosci 27:6473-6477.

Harrison KH, Hof PR, Wang SS (2002) Scaling laws in the mammalian neocortex: does form provide clues to function? J Neurocytol 31:289-298.

Kang J, Jiang L, Goldman SA, Nedergaard M (1998) Astrocyte-mediated 
potentiation of inhibitory synaptic transmission. Nat Neurosci 1:683-692.

Kettenmann H, Verkhratsky A (2008) Neuroglia: the 150 years after. Trends Neurosci 31:653-659.

Nagelhus EA, Mathiisen TM, Ottersen OP (2004) Aquaporin-4 in the central nervous system: cellular and subcellular distribution and coexpression with KIR4.1. Neuroscience 129:905-913.

Nimchinsky EA, Gilissen E, Allman JM, Perl DP, Erwin JM, Hof PR (1999) A neuronal morphologic type unique to humans and great apes. Proc Natl Acad Sci U S A 96:5268-5273.

Oberheim NA, Wang X, Goldman S, Nedergaard M (2006) Astrocytic complexity distinguishes the human brain. Trends Neurosci 29:547-553.

Oberheim NA, Tian GF, Han X, Peng W, Takano T, Ransom B, Nedergaard M (2008) Loss of astrocytic domain organization in the epileptic brain. J Neurosci 28:3264-3276.

Ogata K, Kosaka T (2002) Structural and quantitative analysis of astrocytes in the mouse hippocampus. Neuroscience 113:221-233.

Prabhakar S, Noonan JP, Pääbo S, Rubin EM (2006) Accelerated evolution of conserved noncoding sequences in humans. Science 314:786

Ramon y Cajal S (1897) Histology of the nervous system of man and verte- brates. Reprint (Swanson N, Swanson LW, translator). Oxford: Oxford UP, 1995.

Retzius G (1894) Die neuroglia des Gehirns beim Menschen und bei Saeugethieren. Biol Untersuchungen 6:1-28.

Simard M, Nedergaard M (2004) The neurobiology of glia in the context of water and ion homeostasis. Neuroscience 129:877-896.

Tian GF, Azmi H, Takano T, Xu Q, Peng W, Lin J, Oberheim N, Lou N, Wang X, Zielke HR, Kang J, Nedergaard M (2005) An astrocytic basis of epilepsy. Nat Med 11:973-981.

Volterra A, Meldolesi J (2005) Astrocytes, from brain glue to communication elements: the revolution continues. Nat Rev Neurosci 6:626-640.

Wang SS, Shultz JR, Burish MJ, Harrison KH, Hof PR, Towns LC, Wagers MW, Wyatt KD (2008) Functional trade-offs in white matter axonal scaling. J Neurosci 28:4047-4056.

Wang X, Arcuino G, Takano T, Lin J, Peng WG, Wan P, Li P, Xu Q, Liu QS Goldman SA, Nedergaard M (2004) P2X7 receptor inhibition improves recovery after spinal cord injury. Nat Med 10:821-827.

Yáñez IB, Muñoz A, Contreras J, Gonzalez J, Rodriguez-Veiga E, DeFelipe J (2005) Double bouquet cell in the human cerebral cortex and a comparison with other mammals. J Comp Neurol 486:344-360. 